\title{
Possible role for HIF-1ß/ARNT in the regulation of vascular function in diabetes and cardiovascular disease
}

\author{
Lan $\mathrm{Su}^{2}$, Maura Knapp ${ }^{1}$, Rongxue $\mathrm{Wu}^{1,2^{*}}$ \\ ${ }^{1}$ Section of Cardiology, Department of Medicine, University of Chicago, USA \\ ${ }^{2}$ Department of Cardiology, Wenzhou Medical University, USA
}

\begin{abstract}
Aryl hydrocarbon receptor nuclear translocator (ARNT), also known as hypoxia-inducible factor-1

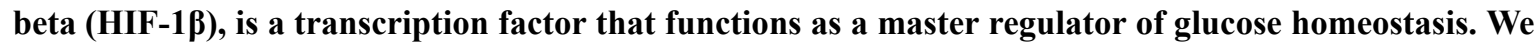
have previously demonstrated that ARNT expression is highest in the heart, where it is required for maintaining normal cardiac metabolism and cardiac function. Diabetes is associated with a loss of ARNT, although the specific role of ARNT in the diabetic heart is still unknown. Diabetes is also characterized by endothelial dysfunction. Thus, investigating the role of endothelial ARNT in diabetic cardiovascular disease could lead to new therapies for treatment or prevention.
\end{abstract}

Accepted on November 15, 2016

\section{Introduction}

Cardiovascular disease is one of the major causes of morbidity and mortality in diabetic patients. In fact, almost $80 \%$ of deaths in diabetics are caused by cardiac complications [1-4]. Because of the high rate of obesity and sedentary lifestyles in the United States, the prevalence of diabetes has doubled over the past 30 years. Diabetes is one of the major risk factors that leads to cardiovascular disease (CVD). In fact, from the perspective of cardiovascular medicine, it may be appropriate to say, "Diabetes is a cardiovascular disease." However, it is still not clear how diabetes promotes cardiac dysfunction. It is known that vascular endothelial cells play a major role in maintaining cardiovascular homeostasis and in patients with diabetes, endothelial dysfunction appears to be a consistent finding. The transcriptional activity of hypoxia-inducible factor- 1 (HIF-1) a heterodimer of HIF- $1 \alpha$ and ARNT (HIF-1 $\beta$ ) - plays a crucial role in the heart's response to ischemia and is essential for glucose metabolism [1]. Regarding the role of HIF-1 in vascular function, HIF- $1 \alpha$ has been intensively studied, but little is known about ARNT (HIF-1 $\beta$ ).

\section{The Link between ARNT and Diabetes}

ARNT was first linked to diabetes in 2005 when Gunton et al. discovered a reduction in ARNT levels in beta cells isolated from patients with diabetes. Out of five genes identified to be important in beta cell function, ARNT was observed to have the most significant impact [3]. ARNT has further been revealed to play a major role in maintaining glucose-stimulated insulin secretion from pancreatic beta cells [5]. Interestingly, a reduction in ARNT levels has also been found in livers isolated from both diabetic human patients and from mouse models of diabetes. Mice with liver-specific ablation of ARNT exhibit features of type 2 diabetes, including increased hepatic gluconeogenesis, increased lipogenic gene expression, and low serum beta-hydroxybutyrate. High glucose and insulin levels, in addition to insulin resistance may be associated with a reduced expression in ARNT in the livers of humans with type 2 diabetes [6]. Consistent with these previous results, we have found a significant reduction in ARNT expression in the hearts of diabetic mouse model, and specific deletion of ARNT leads to a phenotype that mimics diabetic cardiomyopathy. We have identified a role for ARNT as a critical cardiac metabolic mediator that regulates cardiac function through a PPARa pathway [2]. Moreover, our recent studies show a significant reduction in endothelial ARNT in hearts isolated from diabetic animal models, suggesting an important role for endothelial ARNT in the diabetic heart (unpublished data). However, the underlying mechanism in which ARNT may protect endothelial cell function from heart failure in diabetes needs to be further investigated.

\section{Critical Role of ARNT in Vascular Function}

Genetic data have implicated a critical role for HIF-1 $\beta / A R N T$ in vascular function. Targeted disruption of the ARNT gene in a mouse model leads to embryonic lethality between E9.5 and E10.5, mainly due to abnormal vasculature development $[7,8]$. Specific deletion of ARNT in the endothelial cells of mice (Arnt ${ }^{\triangle \mathrm{EC}}$ mice) before the postnatal stage is also lethal, with evidence of liver necrosis, cardiac myocyte degenerative lesion formation, and heart hemorrhage [1]. Interestingly, the effect of disruption of endothelial ARNT on blood vessel formation is restricted only to the liver and heart, which may suggest distinct roles for endothelial ARNT in different organs. Arnt ${ }^{\mathrm{AEC}}$ mice exhibit severe heart hemorrhage with red blood cell accumulation in the myocardium, indicating that ARNT deficiency in endothelial cells leads to disrupted cardiac vessel barrier function and increased vascular leakage. A short-term study on inducible endothelial ARNT deficient mice shows that loss of endothelial ARNT contributes to delayed wound healing, which may be caused by an impaired vasculogenic response [7]. Endothelial dysfunction is mainly characterized by a reduction in the bioavailability of vasodilators, particularly nitric oxide (NO). Our current findings show that deletion of 
Citation: Lan S, Maura K, Rongxue W. Possible role for HIF-1B/ARNT in the regulation of vascular function in diabetes and cardiovascular disease. Curr Trend Cardiol. 2017;1(1):3-4.

ARNT in cultured endothelial cells results in reduced eNOS mRNA levels and a reduction in NO. Thus, these data suggest that ARNT plays an important role in cardiac vascular function.

\section{HIF-1ß/ARNT in Ischemic Heart Disease}

Activation of hypoxia-inducible factor 1 is a critical molecular pathway that functions as a master regulator of oxygen homeostasis. ARNT, as the beta subunit of HIF1, is required for its activation. Activated HIF1a dimerizes with ARNT, generating a functional heterodimer that controls the expression of multiple angiogenic factors. Clinical trials have been initiated to determine the efficacy of gene therapy using angiogenic growth factors in myocardial ischemia [8]. We have observed impaired insulin-stimulated glucose uptake in ARNT deleted endothelial cells. Various reports have shown that in patients with type 2 diabetes, impaired insulin signaling in endothelial cells reduces glucose uptake by the skeletal muscle and leads to cardiac dysfunction [9,10]. These results indicate that reciprocal relationships between endothelial dysfunction and insulin resistance may be a major mediator of heart failure development in diabetic patients. It is necessary to establish a role for endothelial ARNT in aryl hydrocarbon receptor (AHR) and HIF-1alpha signal transduction in the intact mouse.

\section{Summary}

Taken together, these findings indicate that endothelial HIF-1 $\beta$ / ARNT is critical in maintaining endothelial function. Reduced ARNT levels in diabetes may contribute to cardiac heart failure and increased mortality in diabetes. Thus, the endothelial HIF-1 $\beta$ /ARNT pathway may be a therapeutic target for the prevention and treatment of cardiovascular disease in diabetes

\section{References}

1. Maltepe E, Schmidt JV, Baunoch D, Bradfield CA, Simon MC. Abnormal angiogenesis and responses to glucose and oxygen deprivation in mice lacking the protein ARNT. Nature 1997; 386: 403-407.

2. Wu R, Chang HC, Khechaduri A, Chawla K, Tran M, Chai X, Wagg C, Ghanefar M, Jiang X, Bayeva M, Gonzalez F, Lopaschuk G, Ardehali H. Cardiac-specific ablation of ARNT leads to lipotoxicity and cardiomyopathy. J Clin Invest 2014; 124: 4795-4806.
3. Gunton JE, Kulkarni RN, Yim S, Okada T, Hawthorne WJ, Tseng YH, Roberson RS, Ricordi C, O'Connell PJ, Gonzalez FJ, Kahn CR. Loss of ARNT/HIF1beta mediates altered gene expression and pancreatic-islet dysfunction in human type 2 diabetes. Cell 2005; 122: 337-349.

4. Morrish NJ, Wang SL, Stevens LK, Fuller JH, Keen H. Mortality and causes of death in the WHO Multinational Study of Vascular Disease in Diabetes. Diabetologia 2001; 44 Suppl 2: p. S14-21.

5. Pillai, R, Huypens P, Huang M, Schaefer S, Sheinin T, Wettig SD, Joseph JW. Aryl hydrocarbon receptor nuclear translocator/hypoxia-inducible factor-1 \{beta\} plays a critical role in maintaining glucose-stimulated anaplerosis and insulin release from pancreatic \{beta\}-cells. J Biol Chem 2011; 286: p. 1014-24.

6. Wang XL, Suzuki R, Lee K, Tran T, Gunton JE, Saha AK, Patti ME, Goldfine A, Ruderman NB, Gonzalez FJ, Kahn CR. Ablation of ARNT/HIF1beta in liver alters gluconeogenesis, lipogenic gene expression, and serum ketones. Cell Metab 2009; 9: 428-439.

7. Han, Y, Tao J, Gomer A, Ramirez-Bergeron DL. Loss of endothelial-ARNT in adult mice contributes to dampened circulating proangiogenic cells and delayed wound healing. Vasc Med, 2014. 19: p. 429-41.

8. Mercadier JJ, Logeart D. Myocardial gene therapy. Arch Mal Coeur Vaiss 2002; 95: 197-203.

9. Dormishian M, Turkeri G, Urayama K, Nguyen TL, Boulberdaa M, Messaddeq N, Renault G, Henrion D, Nebigil CG. Prokineticin receptor-1 is a new regulator of endothelial insulin uptake and capillary formation to control insulin sensitivity and cardiovascular and kidney functions. J Am Heart Assoc, 2013. 2: p. e000411.

10. Hsieh PC, Davis ME, Lisowski LK, Lee RT. Endothelialcardiomyocyte interactions in cardiac development and repair. Annu Rev Physiol 2006; 68: 51-66.

\section{*Correspondence to}

Rongxue $\mathrm{Wu}$

Department of Cardiology

Wenzhou Medical University

USA 